\title{
Laboratory validation of MEMS-based sensors for post-earthquake damage assessment
}

\author{
Matteo Pozzi ${ }^{\mathrm{a}}$, Daniele Zonta ${ }^{*}$, Juan Santana ${ }^{\mathrm{b}}$, Mikael Colin ${ }^{\mathrm{c}}$, Nicolas Saillen ${ }^{\mathrm{d}}$, Tom Torfs ${ }^{\mathrm{e}}$, \\ Angelos Amditis ${ }^{\mathrm{f}}$, Matthaios Bimpas ${ }^{\mathrm{f}}$, Yorgos Stratakos ${ }^{\mathrm{g}}$, Dumitru Ulieru ${ }^{\mathrm{h}}$, Dimitirs Bairaktaris ${ }^{\mathrm{i}}$, \\ Stamatia Frondistou-Yannas ${ }^{j}$, Vasilis Kalidromitis ${ }^{k}$ \\ ${ }^{a}$ University of Trento, via Mesiano, 77, I-38123, Trento, Italy \\ ${ }^{\mathrm{b}}$ Imec-Holst Centre, HTC, 5605 KN Eindhoven, The Netherlands \\ ${ }^{\mathrm{c}}$ MEMSCAP, Grenoble, France - Corporate R\&D, Parc Activillage des Fontaines, Bernin, France \\ ${ }^{\mathrm{d}}$ Thermo Fisher Scientific, Enschede, The Netherlands \\ ${ }^{\mathrm{e}}$ IMEC, Kapeldreef 75 - 3001 Leuven - Belgium \\ ${ }^{\mathrm{f}}$ National Technical University of Athens - 9, Iroon Polytechniou Str. GR-15773, Athens, Greece \\ ${ }^{g}$ Advanced Microwave Systems Ltd., 25, Martiou Street, 17778, Athens, Greece \\ ${ }^{\mathrm{h}}$ SITEX 45 SRL - 114, Ghica Tei Blvd., Bucharest, Romania \\ ${ }^{\mathrm{i}}$ Bairaktaris and Associates Ltd., 17 Pesmazoglou St., Kiffisia, 145 61, Athens, Greece \\ ${ }^{j}$ RISA Sicherheitsanalysen GmbH - Krumme Straße 55, 10627 Berlin-Charlottenburg, Germany \\ ${ }^{\mathrm{k}}$ TECNIC S.p.A., Via Panama 86/A 00198, Rome, Italy
}

\begin{abstract}
The evaluation of seismic damage is today almost exclusively based on visual inspection, as building owners are generally reluctant to install permanent sensing systems, due to their high installation, management and maintenance costs. To overcome this limitation, the EU-funded MEMSCON project aims to produce small size sensing nodes for measurement of strain and acceleration, integrating Micro-Electro-Mechanical Systems (MEMS) based sensors and Radio Frequency Identification (RFID) tags in a single package that will be attached to reinforced concrete buildings. To reduce the impact of installation and management, data will be transmitted to a remote base station using a wireless interface. During the project, sensor prototypes were produced by assembling pre-existing components and by developing ex-novo miniature devices with ultra-low power consumption and sensing performance beyond that offered by sensors available on the market. The paper outlines the device operating principles, production scheme and working at both unit and network levels. It also reports on validation campaigns conducted in the laboratory to assess system performance. Accelerometer sensors were tested on a reduced scale metal frame mounted on a shaking table, back to back with reference devices, while strain sensors were embedded in both reduced and full-scale reinforced concrete specimens undergoing increasing deformation cycles up to extensive damage and collapse. The paper assesses the economical sustainability and performance of the sensors developed for the project and discusses their applicability to long-term seismic monitoring.
\end{abstract}

Keywords: MEMS, Wireless sensors, Accelerometers, Strain Gauges, Damage Assessment, Long-term monitoring.

\footnotetext{
*daniele.zonta@unitn.it; phone+39 0461 282537; fax +39 0461 282505; http://www.ing.unitn.it/ dzonta
}

Sensors and Smart Structures Technologies for Civil, Mechanical, and Aerospace Systems 2011, edited by Masayoshi Tomizuka, Chung-Bang Yun, Victor Giurgiutiu, Jerome P. Lynch, Proc. of SPIE Vol. 7981, 79810Y (C) 2011 SPIE · CCC code: 0277-786X/11/\$18 - doi: 10.1117/12.882015 


\section{INTRODUCTION}

Structures that are subject to the effects of material aging, design error, overloading, unforeseen foundation settlement or seismic events can progressively accumulate damage during their operational life. Periodical assessment is therefore a key step in rationally planning the maintenance needed to guarantee an adequate level of safety and serviceability.

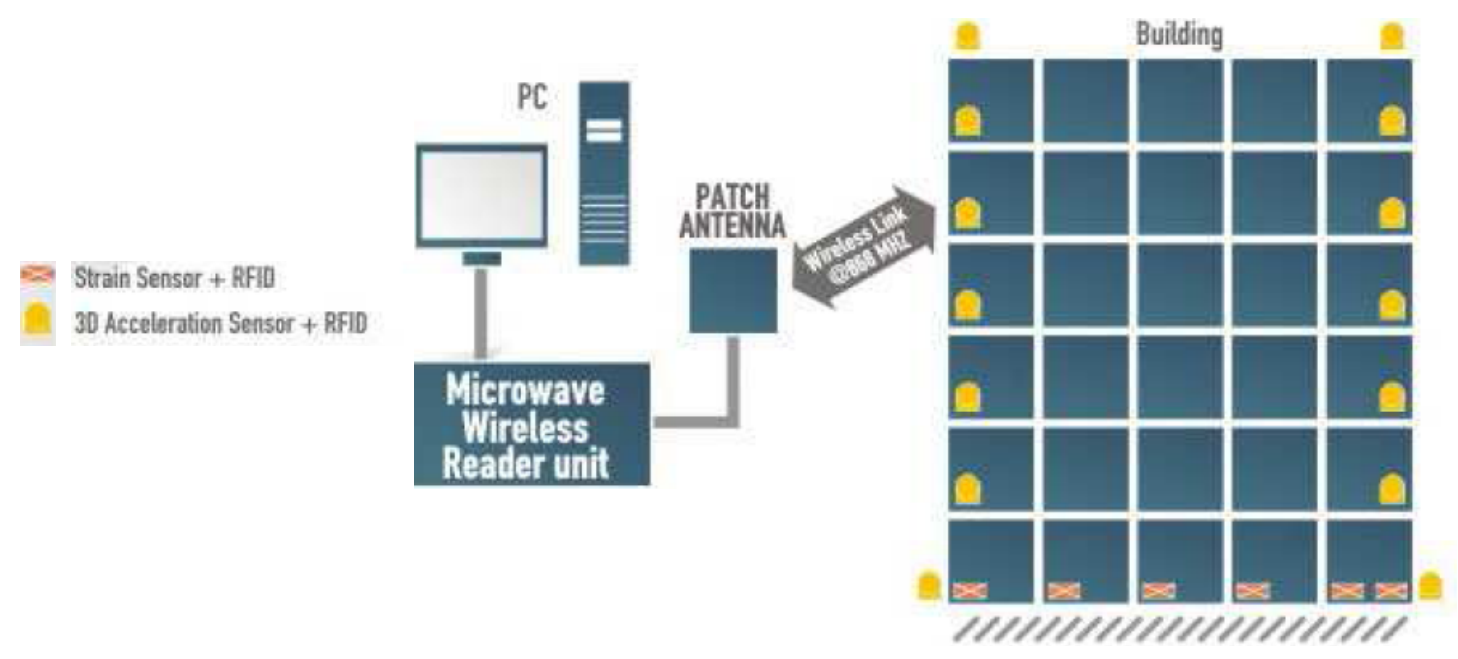

Figure 1. Scheme of the monitoring system proposed in the MEMSCON project.

Nonetheless the economies made available by installing permanent sensing systems are not easy to demonstrate ${ }^{1}$ : the only way to provide sustainability is to make use of durable and robust sensors, industrially produced, which can be easily installed during construction and can furnish information highly relevant to reliability assessment. Modern buildings tend to be overcrowded with electric wiring for power and signals, and the structural monitoring network must not interfere with these: nodes should be small and links wireless. Radio Frequency Identification (RFID) and MicroElectro-Mechanical-System (MEMS) technologies offer a set of promising tools to develop a cost-effective system able to fulfill these requirements. The capability of MEMS and wireless networking for monitoring civil structures is well documented in recent work ${ }^{2,3,4}$. The MEMSCON Project (www.memscon.com) co-funded by the European Community in the 7th Framework Program, started in October 2008 and will last three years, involving partners from seven countries. With this background, the project is devoted to development of a reliable and cost-efficient monitoring system to be integrated in new Reinforced Concrete (RC) buildings for their protection against seismic events and settlement. The system includes a wireless network installed within the building and a base station linking the building to a remote centre for data interpretation. As outlined in Figure 1, the network includes sensing nodes measuring strain and acceleration. Strain measurements are collected at the lowest level of the building, to estimate the vertical column loads and any variation due to settlement; horizontal acceleration is measured by dedicated nodes at each level during an earthquake, allowing analysis of the seismic response of the whole structure.

The research project has two main tasks: (i) development of the sensing network and (ii) development of software for remote data processing, structure condition assessment and for maintenance planning. The products will be validated both in the laboratory and in on-site applications. Task (i) includes creation of new dedicated instruments for strain and acceleration measurement. Both these devices will be based on RFID in MEMS ${ }^{5}$, in small-size packages and with ultralow power consumption. These nodes have been designed to improve their sensing performance with respect to that of existing technology ${ }^{6}$.

The definitive nodes have been produced and are now (December 2010) under laboratory validation; while prototypes were assembled from components available on the market, with the necessary design, packaging and programming. These devices do not now fulfill the target requirements, but they let us investigate the relevant features of the system. The prototypes were tested in the laboratory and this paper reports on that stage of the project. 


\section{STRAIN SENSOR WIRELESS NETWORK}

\subsection{Hardware development}

The Strain Sensor Wireless Network is designed to assess settlement and plastic hinge activation during an earthquake. Sensors will be placed directly on steel reinforcement bars, at the corners of each building column.

At this phase of the MEMSCON project, the strain sensors employed are foil gauges available off-the-shelf, produced by HBM GmbH. Since the gauge is the only component permanently buried in the concrete, the external electronic interrogation node is connected via a 2-wire cable with an appropriate connector. This battery-operated device was designed to partially fulfill the basic MEMSCON specifications: ultra low power consumption (no battery replacement ideally for the 10-year product life), accurate measurement of strain ( $20 \mu \varepsilon$ resolution), and transmission of measurements via a wireless network to the base station.

With this target in mind, the hardware developed for the node includes an analog input management subsystem, with a strain gauge bridge, a programmable amplifier with digital offset and gain adjustments and a low pass filter: when properly calibrated, this subsystem produces a voltage output accurately proportional to the strain applied to the sensor. Data transmission is by a Zigbee Wireless Rx/Tx module, and the node also has a microprocessor for global device control, conversion of analog strain measurements to digital format and a clock that operates continuously for current date and time with $1 \mathrm{sec}$ resolution ${ }^{7}$.

Special firmware was developed that allows the device to wake from sleep-mode at specified time intervals, connect to the RF network, take strain measurements and transmit them to the interrogator; then returning to sleep-mode. The time interval is expected to be $2-4$ months in a practical application, to keep battery power consumption at very low levels. In sleep-mode, the device consumes only $54 \mu \mathrm{W}$, while when active the demand is $100 \mathrm{~mW}$. The electronic system, battery and input connector are all in a plastic box size 11x8x4cm, with an antenna, as illustrated in Figure 2(a).

The base station, shown in Figure 2(b), assigns addresses to the sensing nodes, continuously waits and gives network access to any sensor that asks to transmit data. The acquired data can then be passed to the remote interrogation centre using a 4-element patch antenna array, designed to guarantee a high RF gain to the system. Simulation and validation of the manufactured antenna shows that a reliable link can be expected at up to $1 \mathrm{~km}$ distance in an urban environment when there is line-of-sight contact.

a)

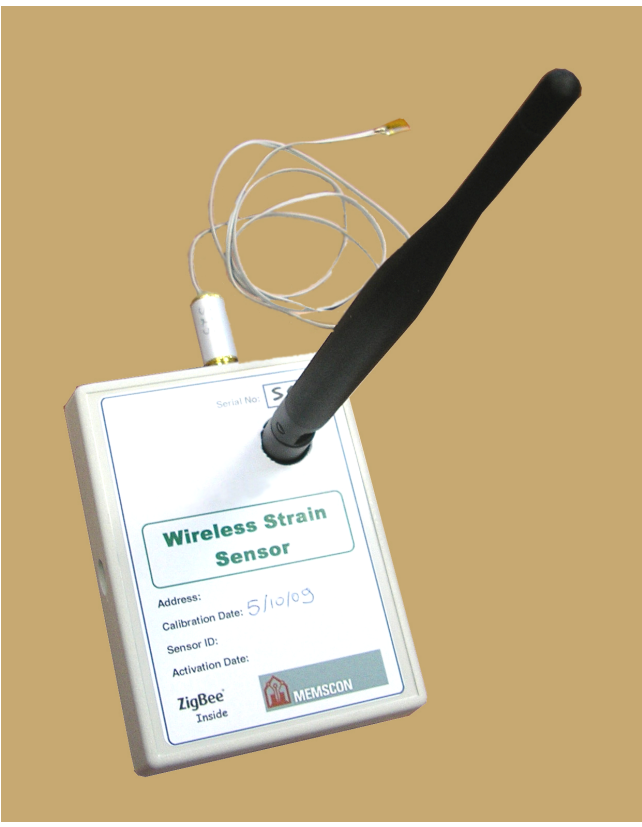

b)

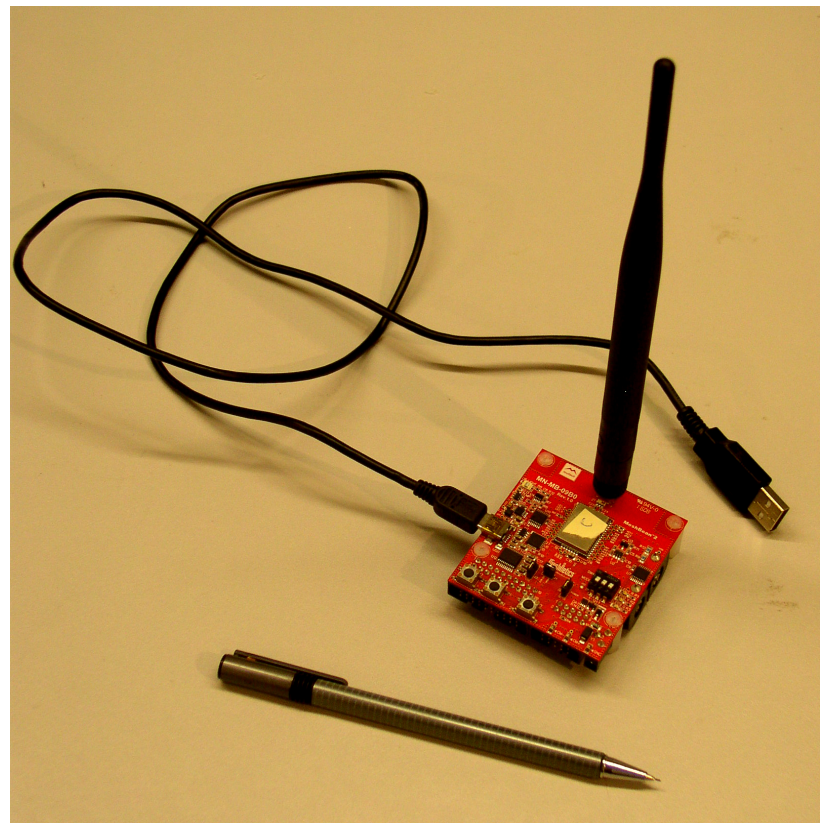

Figure 2. Strain Sensor Node (a) and Base Station (b). 


\subsection{Laboratory validation on small specimens}

The validating campaign for the strain-gauge nodes aims at reproducing in the laboratory the operational conditions of an RC element in a building, up to an extreme scenario. During settlement and, more often, during an earthquake, the columns can undergo extensive damage including concrete cracks, cover spall, crushing and reinforcement yield. As we use the wireless network to estimate the damage level during or after these events, we must investigate the network performance and efficiency under the same conditions.

The gauges were attached to ordinary reinforcing bars (steel B450C, 20mm diameter), with a standard cyanoacrylate adhesive and a silicone protective coating. The installation procedure follows the instructions by HBM, and so the time required to fasten a gauge is about 30 minutes.

In the first laboratory campaign, gauge performance was investigated in small specimens, tested in tensile and compression load cycles. The bars and the complete specimens were also instrumented with additional strain gauges wired to a high-precision interrogation unit, to compare in real-time the data flow deriving from the wireless network with reference values. We adopted the same model of foil gauge, so that the difference between the performance of the wired and wireless systems depends only on interrogation and data transmission. Before starting the laboratory tests, both the clock and the voltage sensitivity of the wireless nodes were calibrated, and the sampling frequency set to $3 \mathrm{~Hz}$.

The simplest way to check the accuracy of the system is to instrument bare reinforcing bars, not embedded in concrete; this lets us avoid all the uncertainties related to the random behavior of concrete. Therefore two bare bars, $50 \mathrm{~cm}$ long, were tested in tensile cycles of increasing amplitude, up to yield and failure. Each bar was instrumented with 1 wired and 2 wireless and gauges, as shown in Figure 3 (upper graph). The same figure reports the outcome of a full test: the strains recorded by the wireless (continuous lines) and by the wired system (dashed line) are plotted against time. The agreement between the two is remarkable, and can be quantified in $20 \mu \varepsilon$, of the same order as the resolution of the wireless system. Note that, independently of the acquisition mode (wired or wireless), the gauges become inactive at a strain of about $2300 \mu \varepsilon$, which is a predictable value for the fatigue life of a glued sensor.

To investigate the performance of the gauges in concrete, three instrumented reinforcing bars were embedded in a concrete cylinder (length: $50 \mathrm{~cm}$; diameter: $13 \mathrm{~cm}$ ). The sensors were placed at the middle of the bar and protected by a Butyl rubber sealant with aluminum foil. When the concrete was poured, the mould was weakened, to induce a crack exactly at the instrumented cross section. The specimens were also instrumented with external sensors to measure the crack opening. Figure 3 (central graph) reports the strain time history, as for the previous test type. Two wireless and two wired gauges were arranged along the same cross section, where a crack is expected. The test shows that the sensors keep working up to the same level of strain as that applied to the bare bar, and the difference between the time histories can be easily attributed to the random behavior of concrete under tension. In fact, during the test, asymmetrical cracks appeared along the specimen, which altered the load eccentricity and, consequently caused variation of the strain along the rebar cross section. The evolution of the load eccentricity during the test was consistent with the measurements derived by the external sensors.

The last tests were devoted to analysis of the behavior of the gauges embedded in concrete under compression. Three concrete specimens (length: $33 \mathrm{~cm}$; diameter: $13 \mathrm{~cm}$ ) were produced, reinforced by the instrumented bar surrounded by a spiral stirrup to reproduce the effect of confinement. External long-base strain gauges were also applied to the concrete surface, to record the behavior of the cover during the test. The specimens underwent cycles of increasing magnitude, up to spalling of the cover and crushing of the concrete core. Four gauges were arranged with 1 wired and 1 wireless sensor on both sides of the rebar; figure 3 (lower graph) shows the measurements obtained against time, for both systems. The strain, now in compression, again reaches $2000-2500 \mu \varepsilon$, and the agreement is of the same order as the resolution.

The network was validated with only two nodes working simultaneously, however additional tests show that the network can easily manage many more sensors. The nodes are activated by a switch and continuously record and send measurements to the base station, which is connected to a PC via USB. The measurements were acquired using Microsoft Hyperterminal and post-processed via Mathworks Matlab. The whole system is user-friendly and stores strain data in a database with a timestamp and sensor identification tag. Over the entire campaign, the network had no problem in data acquisition or transmission. To better couple with the needs of in-field applications, the sampling frequency can be also set by the user, and the strain data can be sent on demand. 

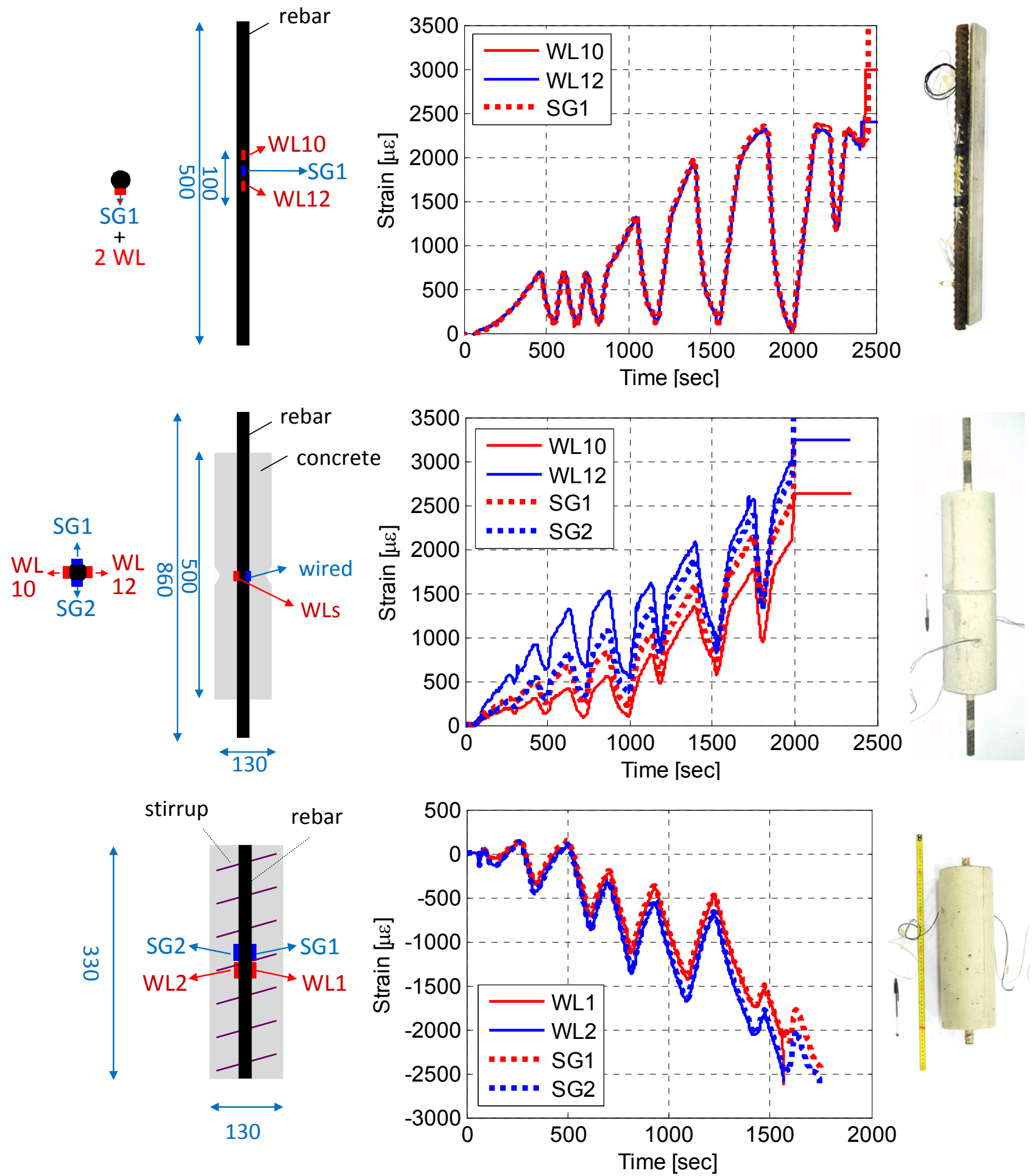

Figure 3. Outcomes of a test on a bare bar (upper graph), a test on a bar embedded in concrete and tensile test (central graph) and a test on an embedded bar tested in compression (lower graph). 

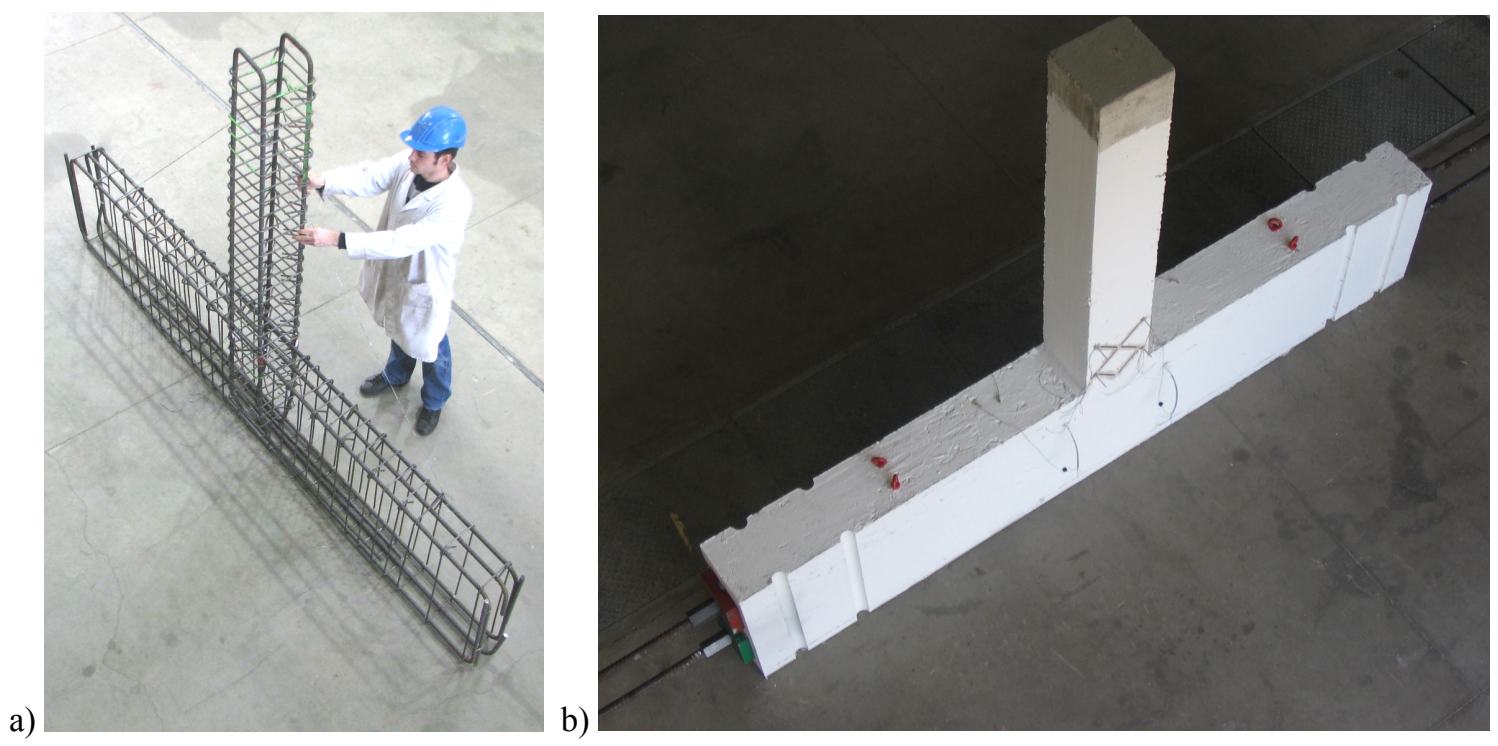

Figure 4. Instrumented reinforcing frame (a) specimen after foundation pre-stressing (b)

\subsection{Laboratory validation on small specimens}

The scope of the second experimental campaign was to extend the results of tests on reduced scale specimens, mentioned above, to a more complex and realistic environment that can simulate the behavior of a real column in a building, up to an extreme scenario. For this purpose, the wireless nodes were tested on three full-scale RC columns, with $300 \mathrm{~mm}$ square section and $1650 \mathrm{~mm}$ height. The specimens were led to collapse by applying horizontal oligo-cyclic displacement of increasing magnitude. Each column was rigidly connected to a massive foundation beam of dimension $3500 \times 700 \times 300 \mathrm{~mm}$, strengthened by pre-stressing and anchored to the strong floor of the laboratory. This set up avoided cracking of the foundation and prevented rotation, and it forced the attivation of a plastic hinge in the column at the interface with the foundation.

The sensors were bonded to the reinforcing bars at the four corners on the base of the column (i.e. at the critical zone), with the same procedure adopted for the previous campaign. Two gauges were bonded to each rebar, one connected to a wireless module, the other to a reference high precision interrogation unit.

After installing the gauges, the reinforcing frame was assembled (figure 4) and the concrete (class C25/30 with maximum inert size $16 \mathrm{~mm}$ and requested fluidity S3) was cast in two stages: the foundation first and the column the day after, to better simulate usual practice. 6 days later, the formwork was removed and the foundation pre-stressed. During the tests, two orthogonal actuators were employed to apply the horizontal displacement and the vertical load. Figure 5 summarizes the final configuration of the tested specimen.

The specimen design resembled that of a column in a standard building in a seismic area. The selected structure is a residential 3-floor building in a seismic zone with ground acceleration $\mathrm{a}_{\mathrm{g}}=2.5 \mathrm{~ms}^{-2}$ on category $\mathrm{B}$ soil according to Eurocode $8^{8}$. It is regular in plan and elevation and the columns, which are pitched at dimension $5 \times 6 \mathrm{~m}$ in plan, are parts of frames connected by $24 \mathrm{~cm}$ thick floors built of brick and concrete. The vertical load on a ground floor column is about $786 \mathrm{kN}$ and the structure is designed in ductility class $\mathrm{M}$, so that the design base shear obtained by an equivalent static seismic analysis is about $105 \mathrm{kN}$ for each column.

Three different loading protocols were applied to each specimen during the laboratory campaign. In the first, only vertical loads were applied cyclically, with increasing amplitude; in the second, a vertical load of $800 \mathrm{KN}$ was applied at the onset, then small lateral displacements were forced, avoiding cracks in the column; in the third and last, the vertical load was applied, followed by the horizontal displacement time history suggested in the ATC-24 standard [9] up to the column failure. In this last protocol, the displacement at which the plastic hinge is activated had been fixed as $\delta_{\mathrm{y}}=8 \mathrm{~mm}$, which underestimates the actual value but let us better investigate the performance of the sensors before their debonding. 


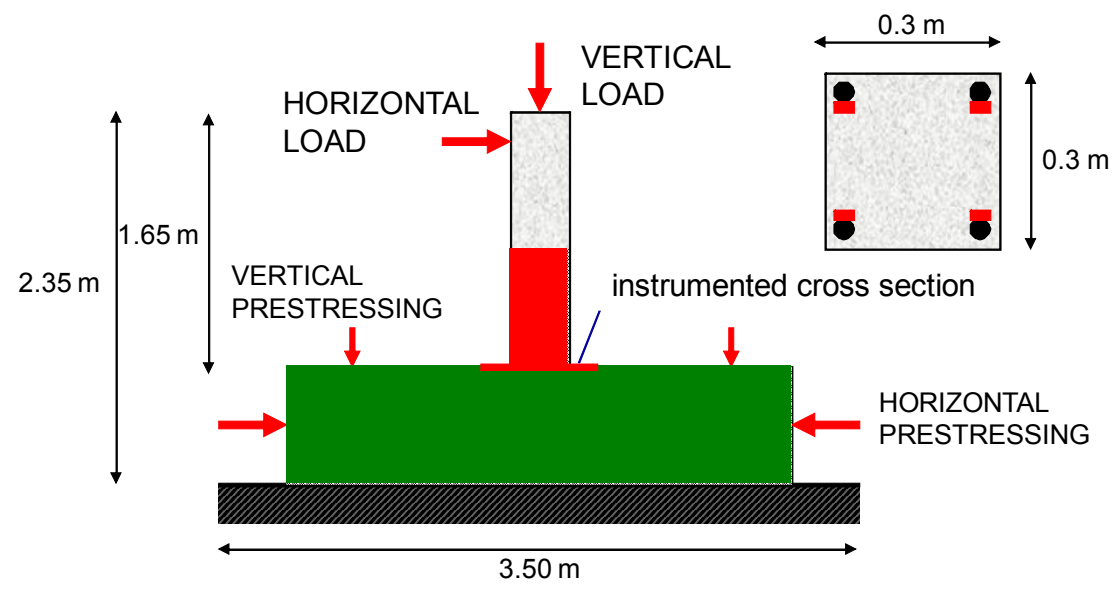

Figure 5. Position of the strain gauges, area where plastic hinge is expected in red, pre-stressed and anchored foundation in green.
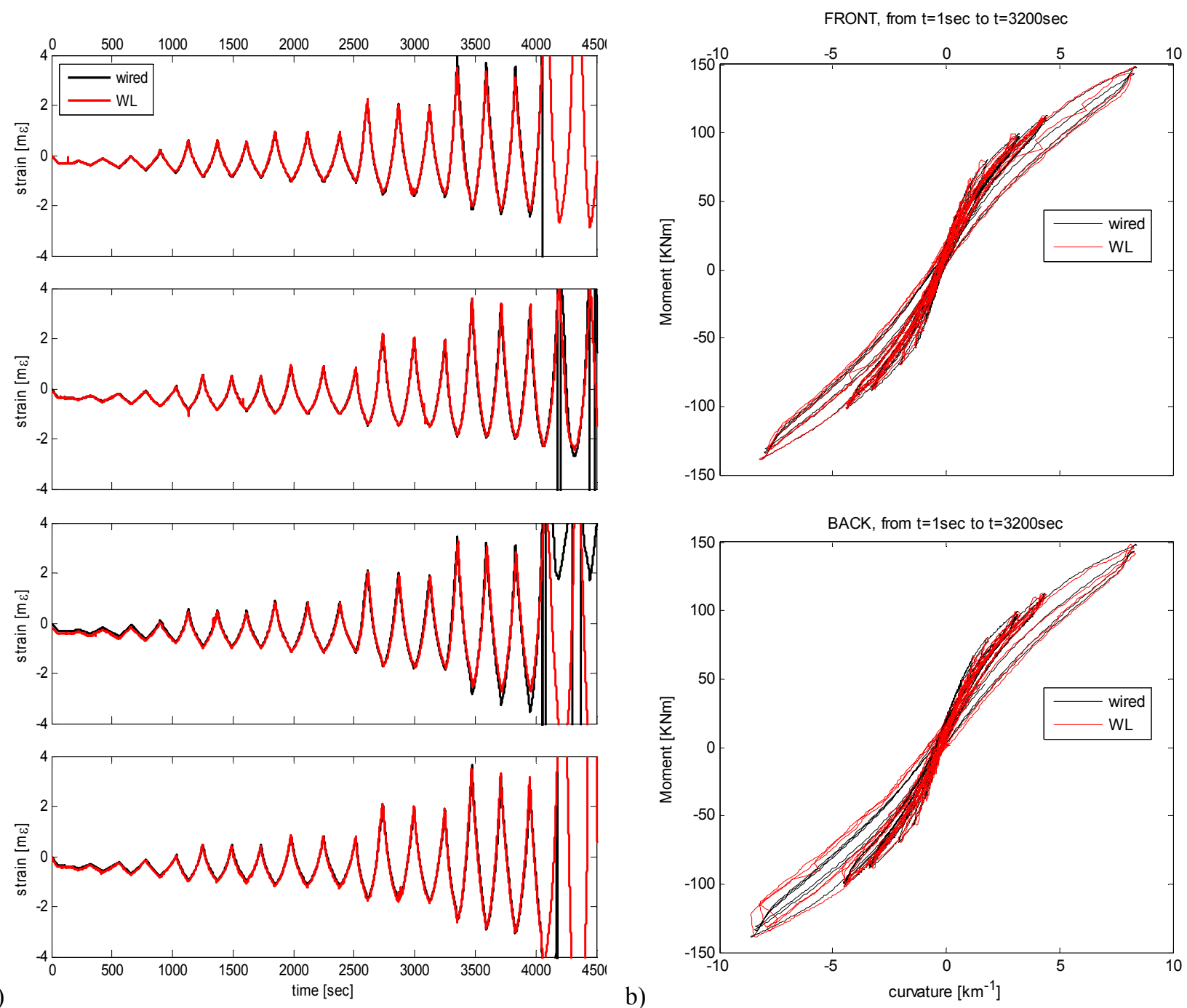

Figure 6. Time history for the strain gauges on the four rebars (a); comparison of moment - curvature graphs for wired and wireless sensors (b). 
Figure 6(a) reports the time histories recorded by the wireless and wired gauges during a destructive test, while figure 6(b) shows the Moment-Curvature diagrams curves calculated with the two acquisition systems on both sides of the column. It is apparent that agreement is very good, and it can be quantified as an RMS error of around $50 \mu \varepsilon$.

This error is probably due to the following causes: some small mechanical differences between foil gauges were expected, the sensors are installed on differing sections at a distance of $13 \mathrm{~mm}$ from each other, and this could be relevant because of the random behavior of concrete.

Despite these differences, the results are still acceptable, as the error is of the same magnitude as the resolution. The error concerning axial deformation of the bars is about $1 \%$, considering that the strain gauges were destroyed at about $4000 \mu \varepsilon$.

\section{ACCELEROMETER WIRELESS NETWORK}

\subsection{Development of the hardware}

The accelerometers will be placed on each level of the building to be monitored, recording horizontal vibration in both orthogonal directions as well as torsion components. The network will be automatically activated during an earthquake, so that it can follow completely the seismic response and consequently estimate damage.

Each accelerometer node measures 3-axis acceleration data, and records and transmits the data over the RF network, using a MEMS-based sensor commercially available from Analog Devices Inc. ${ }^{10}$. This is a completely digital output device that communicates with the system through an SPI interface having a serial EPROM memory and a micro vibration sensor. The firmware developed allows device wake-up upon activation of the micro vibration sensor, fast setup of the node (accelerometer and Zigbee transceiver) from the microprocessor, and data recording in the EPROM. The power consumption of the unit is similar to the strain sensor unit. The micro sensor acts as hardware trigger that activates the node only when substantial vibration (above $20 \mathrm{mg}$ ) occurs. Below this level, the node remains dormant.

To reduce energy consumption, special algorithms were also developed to minimize unit activation by false alarms (vibration caused by events other than earthquakes). Once activated by sufficiently strong vibration, the node acquires acceleration data for a maximum period of 30 seconds, estimating every 5 seconds the amplitude of the vibration along each of the 3 axes. Only if that amplitude is significant (according to a software threshold defined by the user) does the node consider the signal, transmitting data to the base station. The combination of these hardware and software thresholds minimizes the power consumption of the node, avoiding transmission of signals that are irrelevant for seismic analysis: a battery lifespan of years can be expected.

\subsection{Laboratory validation}

To test the performance of the network, three wireless nodes were mounted on a shaking table, back to back with high precision wired piezoelectric seismic accelerometers. The nodes underwent several vibration tests, with excitation of various shape, frequency and amplitude. The aim of the campaign was to characterize the dynamic response of the wireless sensing system and to investigate its accuracy and reliability under conditions similar to those experienced in the field during a seismic event.

Two types of test were performed: in the "calibration tests", the nodes were mounted in parallel on the table, and each axis was tested with harmonic excitation at frequencies in the range $1-20 \mathrm{~Hz}$, at amplitude ranging from $+/-1 \mathrm{~ms}^{-2}$ to $+/-$ $4 \mathrm{~ms}^{-2}$. This sequence allowed calibration of the sensitivity of each node axis and tuning of the internal clock. The resulting response of the wireless network has a maximum discrepancy respect to the wired system of the order of 3\%.

Then, to better simulate operational conditions, the wireless sensors were mounted on a two-storey metal frame fixed to the shaking table, again back to back with the wired instruments, as shown in figure 7 (lower picture). One sensor was placed on the table, to record the "ground" vibration, and the other two at the upper floors, to measure the frame response. We excited the frame both with frequency-sweeps and with seismic-like waves. Figure 7 (upper graph) shows the comparison between the vibrations recorded by the wired and the wireless systems, at each floor, during a sweep. Agreement is very good, of the same order as the resolution of the wireless system, which is $18 \mathrm{mg}\left(=0.18 \mathrm{~ms}^{-2}\right)$.

This type of test also allows an estimate of the modal properties of the metal frame (the reader is referred to the book by Ewins $^{11}$ for information on the fundamentals of modal analysis), which in turn reproduce the mechanical response 
expected in a full scale building undergoing an earthquake. The lower graph of figure 7 reports the Frequency Response Functions of each floor to the ground excitation, as estimated by the wired and wireless sensors. This graph allows identification of the resonance and anti-resonance frequencies of the structure, which remain linear during the tests. Here again the two signals are in good agreement except below the $2 \mathrm{~Hz}$ where the wireless sensors exhibit a high level of noise.

The network easily manages several nodes working simultaneously, because the recorded vibration only lasts a maximum time of half a minute. As for the strain gauge nodes, the accelerometer node is activated by a switch, and measurement acquisition is automatically started when the node moves. In the configuration adopted during the laboratory campaign, the data are stored in a database, with timestamp, sensor and axis identification tags, using Hyperterminal and Matlab. A limit of the present configuration of the sensing network is that the activation criterion acts locally on each node, thus in general the whole network does not wake up simultaneously.

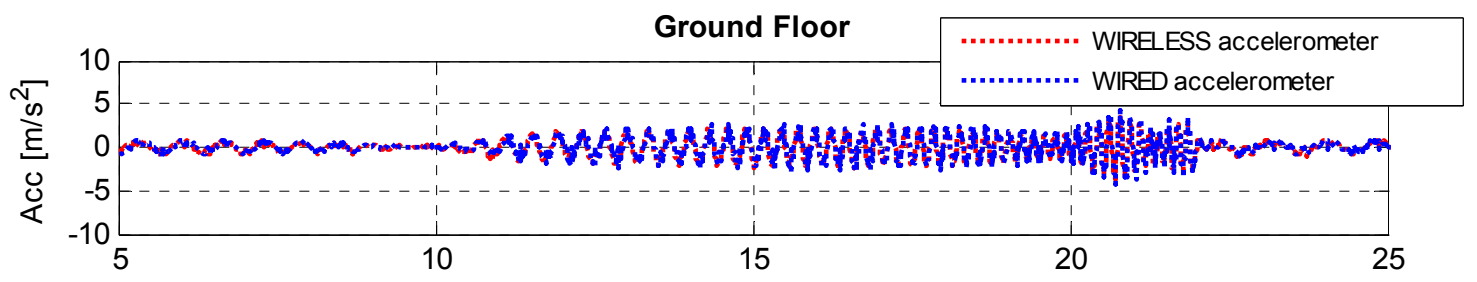

First Floor
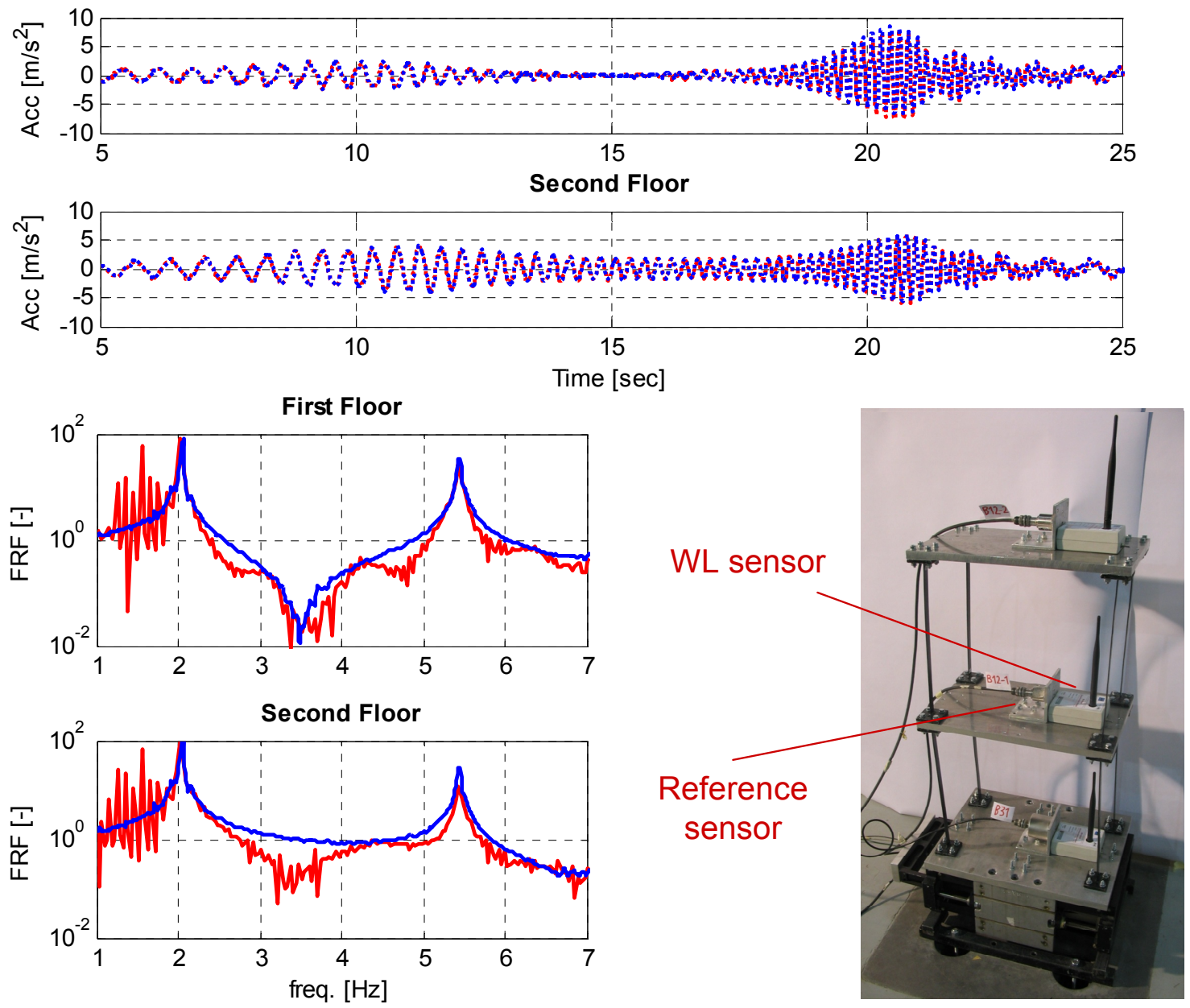

Figure 7. Example of a frequency-sweep test mounting the sensors on the metal frame: time histories (upper graph), frequency response functions (lower graph) and appearance of the instrumented metal frame on the shaking table (lower picture) 


\section{CONCLUSIONS}

Both the strain gauge and the accelerometer networks proved to be reliable under the operational conditions expected in a field application. The performance in strain measurement allowed us to follow the onset of yield in the reinforcing steel, which is usually a limitation for sensors applied to civil structures. This is suitable for assessment of settlement. In the next few months, additional tests will be done on full-scale column specimens, to investigate the behavior of a larger wireless network in a more complex environment and in a seismic scenario.

The accelerometer network proved to be reliable in the frequency range relevant to seismic analysis, with sufficient accuracy, and several tri-axial nodes can be employed simultaneously. Data acquisition is triggered automatically when a vibration threshold is exceeded, and this allows long-term monitoring, recording all relevant seismic events.

The final products of the MEMSCON project, now available, try to improve on this performance. In particular, the packaging size has been reduced, the measurement range improved and the power consumption reduced. Further development of the accelerometer nodes will be related to resolution improvement, to real-time synchronization of the nodes to allow acquisition of a reliable time-stamp years after installation, and adoption of a more sophisticated algorithm to reduce the number of false alarms and cause simultaneous wake up of the whole network.

The measurements provided by the sensing networks are conceived for post-processing by a Decision Supporting System (DSS) which will automatically assess the condition state and suggest an optimal maintenance strategy for the building. This DSS and its interface with the measurement flow are now under development.

\section{ACKNOWLEDGMENTS}

The MEMSCON project is funded by the European Committe with Grant Agreement n. CP-TP 212004-2. The authors wish to thank all MEMSCON partners and particularly all those involved in the development of this phase of the project. The contribution of Davide Dal Bosco, Matteo Accorti, Sadri Mevel and Stavros Tsakirhs to the experiments reported in this paper is especially acknowledged.

\section{REFERENCES}

[1] Pozzi, M., Zonta, D., Wang, W. and Chen, G., "A framework for evaluating the impact of structural health monitoring on bridge management", Proc. IABMAS 2010, (2010).

[2] Lynch, J.P. and Loh, K.J., "A summary review of wireless sensors and sensor networks for structural health monitoring", The shock and vibration digest 38(2), (2006).

[3] Zonta, D., Pozzi, M., Zanon, P., "Managing the Historical Heritage Using Distributed Technologies", International Journal of Architectural Heritage 2, 200-225 (2008).

[4] Kruger, M., Grosse, C.U. and Marron, P.J., "Wireless Structural Health Monitoring Using MEMS", Key Engineering Materials 293-294, 625-634 (2005).

[5] Yusuke, I., Suzuki, S., Okamoto, H., Murakami, H., Asama, S., Morishita, S., Mishima, T., Lin, X. and Itoh, H., "Force sensor system for structural health monitoring using passive RFID tags", Sensor Review 29(2), 127-136 (2009).

[6] Ferrar, C.R., Park, G., Allen, D.W. and Todd, M.D., "Sensor Network Paradigms for Structural Health Monitoring", Journal of Structural and Health Monitoring 13(1), 210-225 (2006).

[7] Jiang, X.D, Tang, Y.L. and Lei, Y., "Wireless sensor networks in Structural Health Monitoring based on ZigBee technology", Proc. of ASID Conference 2009, 449-452 (2009).

[8] CEN, [Eurocode 8: Design of structures for earthquake resistance. Part 1-1: General rules, seismic actions and rules for buildings], European Committee for Standardization, (2004).

[9] ATC, [ATC-24: Guidelines for cyclic seismic testing of components of steel structures], (1992).

[10] Selvarajan, A., "MEMS and photonics technologies for structural health monitoring." SPIE: Smart Materials, Structures, and Systems, 12-14 December, Bangalore, India, Vol.5062, 10-17 (2003).

[11] Ewins, D.J., [Modal Testing], Wiley, (2001). 